\title{
Line of Sight Robot Navigation Toward a Moving Goal
}

\author{
Fethi Belkhouche, Member, IEEE, Boumediene Belkhouche, Member, IEEE, and \\ Parviz Rastgoufard, Senior Member, IEEE
}

\begin{abstract}
In this paper, we consider the problem of robot tracking and navigation toward a moving goal. The goal's maneuvers are not a priori known to the robot. Thus, off-line strategies are not effective. To model the robot and the goal, we use geometric rules combined with kinematics equations expressed in a polar representation. The intent of the strategy is to keep the robot between a reference point, called the observer, and the goal. We prove under certain assumptions that the robot navigating using this strategy reaches the moving goal successfully. In the presence of obstacles, the method is combined with an obstacle avoidance algorithm. The robot then moves in two modes, the navigation mode and the obstacle avoidance mode. Simulation of various scenarios highlights the efficiency of the method and provides an instructive comparison between the paths obtained for different reference points.
\end{abstract}

Index Terms-Line of sight guidance law, relative kinematics equations, robotic navigation toward a moving goal, tracking.

\section{INTRODUCTION}

$\mathbf{N}$ AVIGATION toward a moving goal, tracking, and interception of moving objects using wheeled mobile robots is an important field in robotics. Various types of applications may benefit from this field such as autonomous surveillance, where the robot aims to track a moving object and keep it in a surveillance zone. Soccer robotics is another example, where the robot aims to reach the moving ball. The literature on robot navigation is substantial, ranging over various methods, such as potential field methods ([1]-[3]), vector field histogram ([4], [5]), and visual servoing control ([6], [7]). Most of the research is concentrated on stationary goals. When a moving goal is considered, the navigation problem becomes quite difficult, especially when the maneuvers of the goal are unpredictable or unknown to the robot. In this case, the control law must be a real-time strategy. This problem is fairly similar to the problem of tracking and pursuing moving objects using mobile robots. For this purpose, methods based on artificial vision, artificial intelligence, fuzzy control, and nonlinear control were suggested.

There exist mainly two classes of methods used for trackingfollowing and navigation toward moving objects: model-based and feature-based. Model-based methods use a model of the tracked objects. They are more robust in general. Feature-based methods track features such as object contours, colors, region of interest, etc.

Manuscript received September 28, 2004; revised March 21, 2005. This paper was recommended by Associate Editor J. Wang.

The authors are with the Electrical Engineering and Computer Science Department, Tulane University, New Orleans, LA 70118 USA (e-mail: belkhouf@eecs.tulane.edu).

Digital Object Identifier 10.1109/TSMCB.2005.856142
Our work here is motivated by the importance of the topic of real-time robot navigation toward a moving goal. This topic is interesting from both theoretical and practical point of views, since it has a wide range of civilian and military applications (such as soccer robotics and surveillance for example). Unfortunately, little attention is given to this topic compared to stationary goals. We are also motivated by the fact that most of the suggested methods are feature-based methods. These are not as robust as model-based methods and may be computationally expensive.

Different methods are discussed in the literature. Various fuzzy logic approaches for control and decision making are used ([8]-[13]). In [14], a fuzzy logic controller is used to solve the problem of pursuit. In [10], the aim is to control a robot in order to reach another robot. Two fuzzy sliding mode control rules are used for the steering angle and the speed of the robot. In ([8], [13]), the authors use a fuzzy logic look-ahead algorithm for motion control with the Grey algorithm to predict the target position. Genetic learning fuzzy logic control rules to capture the target are discussed in [11]. In [9], fuzzy tracking control of a target using a mobile robot is achieved using infrared sensors, where a fuzzy sliding mode control scheme is suggested to accomplish the control task. Methods based on fuzzy control approaches may suffer from the following drawbacks.

1) Fuzzy control rules must be determined properly for the tracking system to function correctly.

2) Fuzzy control laws are obtained after trial-and-error experiments, which may be time consuming. When significant changes occur outside the experiment settings, the fuzzy controller must be re-tuned.

3) It is difficult to come up with an optimal solution, due the imprecise nature of the controller.

A model-based approach is suggested in [15], where Lyapunov theory is used to derive a stable control law to accomplish the task of tracking a moving target using a unicycle mobile robot. Lyapunov theory combined with potential field methods is used in [16] for fast target pursuit. The difficulty of construction of Lyapunov functions is the major drawback of Lyapunov theory-based tracking methods. Cooperative hunting behavior is addressed in [17] and [18], where the author focuses more on the cooperative behavior than the navigation strategy to catch the prey. The control laws for the robots are based on potential fields methods. In [19], the potential field method is suggested for robot navigation in a dynamic environment. Potential field methods for navigation toward moving goals suffer from the same problem as stationary goals; for example, the robot can be trapped into local minima of the potential function other than the goal configuration. This problem appears more frequently 
in the case of moving goals. Furthermore, the potential function must be updated on-line, which is a difficult task due to the nature of potential functions.

Visual servoing is another family of methods widely used for this purpose. Various methods and strategies based on visual servoing control were suggested for the pursuit ([20]-[28]). Different problems such as real-time implementation ([20], [21]), tracking in the presence of uncertainty [22], tracking of complex objects [23] and compensation for abrupt changes in the target motion [24] were considered. Positioning and localization of a robot with respect of the target is considered in ([25] and [29]), where the task function approach is used for visual servoing in [29]. Freyrer and Zell ([26] and [27]) consider the problem of tracking humans using wheeled mobile robots based on artificial vision. Simulating the pursuit of a moving target using a robot based on artificial vision is considered in [28].

Even though algorithms based on artificial vision seem to be quite efficient, they may suffer from the following problems.

1) Processing the huge amount of data coming from the camera in real time is a difficult problem requiring fast algorithms, especially for fast moving targets. Data reduction is necessary in some situations.

2) The moving target must stay within the camera scope, which requires real-time camera callibration; otherwise, the navigation algorithm fails.

In this paper, our aim is to contribute to the solution of the problem of tracking-interception of a moving object by a wheeled mobile robot and navigation toward a moving goal. The robot aims to reach the moving goal from any initial position. The goal maneuvers are not a priori known to the robot, and therefore, solving the tracking-navigation problem requires a real-time strategy. We model the robot and the goal kinematics equations in polar coordinates to obtain a mathematical model of the tracking problem. Our control strategy is adapted from the line of sight guidance law. This strategy belongs to the family of classical guidance laws, which are based on the integration of the kinematics equations with geometric rules. These laws are well studied in the aerospace community ([30]-[32]). The proportional navigation is among the most discussed guidance law in the literature [33]. The application of different variants of the proportional navigation to solve the problem of robotic arm interception of a moving object is discussed in ([34], [35]), where the robustness and the computational efficiency of the method are shown. In [36], the deviated pursuit, which is a particular case of the proportional navigation is suggested for robot's navigation. The algorithm was integrated with a histogram-like approach. The main difference between the proportional navigation and the line of sight strategies is that the line of sight strategy makes use of the notion of an observer as a control station or reference point. However, no such notion exists in the case of the proportional navigation. Therefore, the proportional navigation is a two-point control law, whereas the line of sight is a three-point control law. Our work is also a contribution to model-based methods used for the purpose of tracking-navigation of moving objects, where we introduce the notion of an observer. Here, the navigation problem toward an unpredictably moving goal is considered in both obstacle-free workspace and in the presence of obstacles.
In the latter case, the robot moves in two modes, the navigation mode and the obstacle avoidance mode.

This paper is organized as follows. In Section II, we formulate the problem. In Section III, we define the robot's model and we introduce important geometric variables. The kinematics equations model for the tracking-navigation problem are derived in Section IV. In Section V we introduce the navigation strategy and we discuss its properties. In Section VI, an obstacle avoidance algorithm is integrated with the method. An extensive simulation for different scenarios is given in Section VII.

\section{Problem Formulation}

The target (the goal) moves with unknown maneuvers in the workspace $W$, which consists of a subspace of $\mathbb{R}^{2}$. The path traveled by the target is denoted by $P_{T}(t)=\left(x_{T}(t), y_{T}(t)\right)$, where $x_{T}$ and $y_{T}$ are the Cartesian coordinates of the target in the world coordinates system $\left\{W_{r}\right\}$ with $O$ as the origin. Similarly, the robot's path is denoted by $P_{R}(t)=\left(x_{R}(t), y_{R}(t)\right)$, where $x_{R}$ and $y_{R}$ are the coordinates of the robot in the Cartesian frame of reference. The purpose is to design a navigation law which allows the robot to reach the moving goal. Thiscanbe expressed as $P_{R}\left(t_{f}\right) \simeq P_{T}\left(t_{f}\right)$ with $t_{f}<+\infty$. $t_{f}$ is called the interception time. It is a function of the goal maneuvers, the initial distance, and the linear velocities of the robot and the target. If we assume that the size of the robot and the target are small enough to be dropped, then, the interception is characterized by $P_{R}\left(t_{f}\right)=P_{T}\left(t_{f}\right)$ (which is an approximation of the realworld). The robot starts moving in the workspace at the initial time $t_{0}$ in order to reach the moving target. We assume that the following conditions are satisfied.

(H1) The robot is faster than its moving goal (target), i.e., $v_{R}>v_{T}$

(H2) The minimum turning radius of the robot is smaller than the minimum turning radius of the target.

(H3) The path of the target is smooth.

As mentioned previously, our aim is to design an on-line navigation law for the robot, which allows the robot to navigate and reach a goal moving unpredictably. We suggest a solution to this problem based on the line of sight guidance law.

\section{RoBot MOdEL AND GEOMETRY OF THE TRACKING PROBLEM}

The robot is a simple wheeled mobile robot of the unicycle type. The kinematics equations of the robot are given by the following model:

$$
\begin{aligned}
& \dot{x}_{R}=v_{R} \cos \theta_{R} \\
& \dot{y}_{R}=v_{R} \sin \theta_{R} \\
& \dot{\theta}_{R}=\omega_{R}
\end{aligned}
$$

where $\theta_{R}$ is the robot orientation angle with respect to the reference line (which is parallel to the $\mathrm{x}$-axis), $v_{R}$ is the robot's linear velocity and $\omega_{R}$ is the angular velocity. The velocities $v_{R}$ and $\omega_{R}$ are also the control inputs. Similarly, the target moves in the Cartesian frame of reference according to the following kinematics equations:

$$
\begin{aligned}
& \dot{x}_{T}=v_{T} \cos \theta_{T} \\
& \dot{y}_{T}=v_{T} \sin \theta_{T}
\end{aligned}
$$




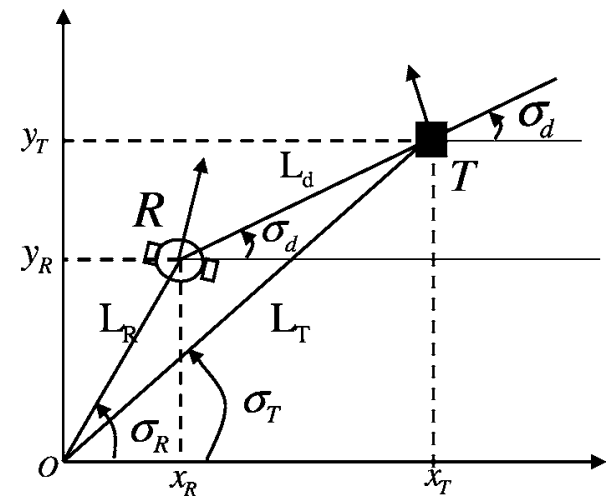

Fig. 1. Geometry of the tracking problem.
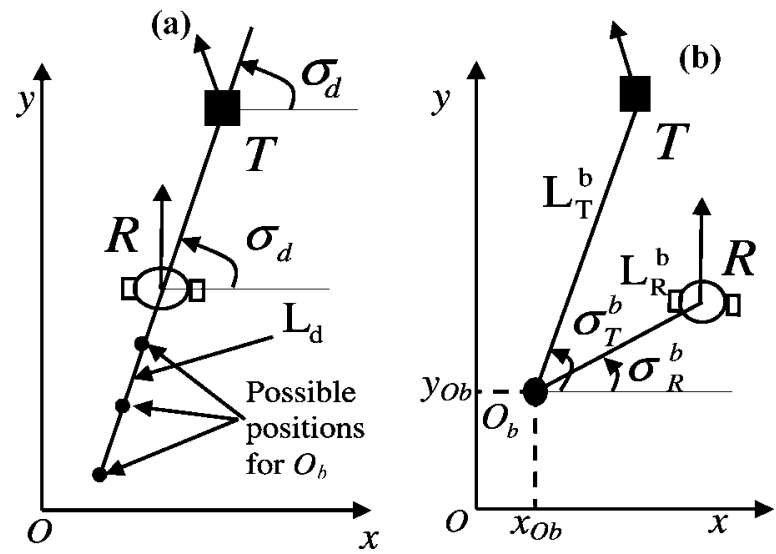

Fig. 2. Geometry of the tracking: (a) illustration of the observer positions that satisfy assumption (H4) and (b) illustration of the range and line of sight angle measured based on the observer.

where $v_{T}$ is the target's linear velocity and $\theta_{T}$ is the target orientation angle with respect to the positive x-axis. $\theta_{T}(t)$ is an unknown function, but it can be measured from a given reference point. For simplicity, we assume that both the target and the robot move with constant speed.

The approach used in this paper is based on the kinematics equations of the robot and the target combined with geometrical rules. Before we discuss the navigation strategy, it is necessary to introduce some definitions for the geometric quantities being used in this paper. Let $O$ be the origin of the world coordinates system. The reference line is represented by the $\mathrm{x}$-axis. Consider Figs. 1 and 2, which illustrate the geometry of the tracking problem. The robot is denoted by $R$ and the goal by $T$. We define the following quantities.

1) The positions of the robot and the target in the reference frame of coordinates are given by $\mathbf{r}_{R}=\mathbf{O R}$ and $\mathbf{r}_{T}=\mathbf{O T}$, respectively. $r_{R}(t)$ is the Euclidean distance between the origin and the robot's reference point and $r_{T}(t)$ is the Euclidean distance between the origin and the target. The velocity vectors are given by $\dot{\mathbf{r}}_{R}$ and $\dot{\mathbf{r}}_{T}$.

2) The imaginary straight line joining the origin of the reference frame and the reference point of the robot is called the robot's line of sight and it is denoted by $\mathbf{L}_{\mathbf{R}}$. In a similar way, the imaginary straight line joining the origin of the reference frame and the target is defined as the target's line of sight and it is denoted by $\mathbf{L}_{\mathbf{T}}$.

3) The imaginary straight line that starts at the robot and is directed toward the target is the line of sight robottarget. This line is denoted by $\mathbf{L}_{\mathbf{d}}$ in Fig. 1.

4) The line of sight angles of the robot and the target are respectively denoted by $\sigma_{R}$ and $\sigma_{T}$. These are the angles from the reference line to the lines of sight of the robot and the target, respectively.

5) The angle of the line of sight robot-target is given by $\sigma_{d}$. This is the angle between the reference line and the line of sight robot-target.

6) The stationary point $O_{b}\left(x_{O_{b}}, y_{O_{b}}\right)$ is defined as the stationary observer. It is assumed that:

(H4) At the initial time $t_{0}$ the target, the robot, and the observer lie on the same straight line, and the robot is between the observer and the target. A particular case is when $O_{b}$ is at the initial position of the robot. An illustration is shown in Fig. 2(a).

This assumption is related to the derivation of the navigation law. The detailed description of the reason behind this assumption is given in Section $\mathrm{V}$ after the introduction of the control law. Under assumption (H4), there exists an infinite number of possibilities for the position of the observer. The following assumption concerns the sensory system.

(H5) The observer has a sensory system that allows to measure the positions of the target and the robot, their linear velocities, and the orientation angle of the target.

7) We also define the lines of sight and the line of sight angles based on the observer point. We denote by $\mathbf{L}_{\mathbf{R}}^{\mathbf{b}}$ the line of sight joining $O_{b}$ and the robot's reference point, and by $\mathbf{L}_{\mathbf{T}}^{\mathbf{b}}$ the line of sight joining $O_{b}$ and the target. The line of sight angles constructed from the reference line to the lines $\mathbf{L}_{\mathbf{R}}^{\mathbf{b}}$ and $\mathbf{L}_{\mathbf{T}}^{\mathbf{b}}$ are denoted by $\sigma_{R}^{b}$ and $\sigma_{T}^{b}$, respectively. The distance observer-robot and the distance observer-target are denoted by $r_{R}^{b}$ and $r_{T}^{b}$, respectively. Under assumption (H4), two important properties are satisfied, namely, the inequality given by

$$
r_{R}^{b}\left(t_{0}\right)<r_{T}^{b}\left(t_{0}\right)
$$

and

$$
\sigma_{R}^{b}\left(t_{0}\right)=\sigma_{R}^{b}\left(t_{0}\right)
$$

The relative distance robot-target is given by

$$
r_{d}=\sqrt{\left(x_{T}-x_{R}\right)^{2}+\left(y_{T}-y_{R}\right)^{2}} .
$$

The ranges $r_{R}$ and $r_{T}$ are expressed in terms of the robot and the target coordinates as follows:

$$
r_{R}=\sqrt{x_{R}^{2}+y_{R}^{2}}
$$

and

$$
r_{T}=\sqrt{x_{T}^{2}+y_{T}^{2}} .
$$


The line of sight angles for the robot and the target can also be expressed in terms of the Cartesian coordinates as follows

$$
\begin{aligned}
\tan \sigma_{R} & =\frac{y_{R}}{x_{R}} \\
\tan \sigma_{T} & =\frac{y_{T}}{x_{T}} .
\end{aligned}
$$

Similarly, we get for the angle of the line of sight robot-target

$$
\tan \sigma_{d}=\frac{y_{T}-y_{R}}{x_{T}-x_{R}}
$$

Note that $\sigma_{d}$ and $\dot{\sigma}_{d}$ are not defined when $r_{d}=0$, i.e., at the interception time.

The moving target can perform two types of motions, namely accelerating and nonaccelerating. In the case of nonaccelerating motion, the target moves with constant speed and constant orientation angle. In the second case, which is more difficult, the target moves with time varying speed or time varying orientation angle, thus non zero angular velocity. Since we are assuming that $v_{T}$ is constant, an accelerating target in this paper refers to a target moving with time-varying orientation angle.

\section{KINEMATICS MODELS}

We use a polar representation to derive kinematics models for the robot and the target. The velocity of the target in the Cartesian frame of reference seen by the robot is given by

$$
\begin{aligned}
& \dot{x}_{d}=v_{T} \cos \theta_{T}-v_{R} \cos \theta_{R} \\
& \dot{y}_{d}=v_{T} \sin \theta_{T}-v_{R} \sin \theta_{R}
\end{aligned}
$$

with $x_{d}=x_{T}-x_{R}$ and $y_{d}=y_{T}-y_{R}$. The robot reaches its goal when $x_{d}$ and $y_{d}$ are equal to zero simultaneously, i.e., $r_{d}=$ $\sqrt{x_{d}^{2}+y_{d}^{2}}=0$. The analysis based on the Cartesian models is difficult. For this reason, we use polar coordinates to facilitate the analysis. We proceed by deriving equivalent models of the robot and the target kinematics equations in polar coordinates. Consider the following change of variable:

$$
\begin{aligned}
& x=r \cos \sigma \\
& y=r \sin \sigma
\end{aligned}
$$

where $r$ and $\sigma$ are the radial and the angular variables, respectively. By taking the derivative of $r$ and $\sigma$ with respect to time, we get

$$
\dot{r}=\frac{\dot{x} x+\dot{y} y}{r}
$$

and

$$
\dot{\sigma}=\frac{\dot{y} x-\dot{x} y}{r^{2}} .
$$

By combining (12) and (13) with (11) and replacing $\dot{x}$ and $\dot{y}$ by their values in the kinematics equations $(\dot{x}=v \cos \theta, \dot{y}=$ $v \sin \theta$ ), we get

$$
\dot{r}=v \cos (\theta) \cos (\sigma)+v \sin (\theta) \sin (\sigma)
$$

and

$$
r \dot{\sigma}=v \sin (\theta) \cos (\sigma)-v \cos (\theta) \sin (\sigma)
$$

These equations are valid for both the robot and the target. The polar kinematics models for the robot and the target are obtained from (14) and (15) easily by using the kinematics equations of the robot and the target and trigonometric identities. We get for the robot

$$
\begin{aligned}
\dot{r}_{R} & =v_{R} \cos \left(\theta_{R}-\sigma_{R}\right) \\
r_{R} \dot{\sigma}_{R} & =v_{R} \sin \left(\theta_{R}-\sigma_{R}\right)
\end{aligned}
$$

and for the moving target

$$
\begin{aligned}
\dot{r}_{T} & =v_{T} \cos \left(\theta_{T}-\sigma_{T}\right) \\
r_{T} \dot{\sigma}_{T} & =v_{T} \sin \left(\theta_{T}-\sigma_{T}\right)
\end{aligned}
$$

where $\dot{r}_{R}$ and $r_{R} \dot{\sigma}_{R}$ are the components of the robot's velocity vector along and across the line of sight $\mathbf{L}_{\mathbf{R}}$, and $\dot{r}_{T}$ and $r_{T} \dot{\sigma}_{T}$ are the components of the target's velocity vector along and across the line of sight $\mathbf{L}_{\mathbf{T}}$. The components of the relative velocity of the target seen by the robot along and across the line of sight $\mathbf{L}_{\mathbf{d}}$ can be obtained as follows:

$$
\begin{aligned}
\dot{r}_{d} & =v_{T} \cos \left(\theta_{T}-\sigma_{d}\right)-v_{R} \cos \left(\theta_{R}-\sigma_{d}\right) \\
r_{d} \dot{\sigma}_{d} & =v_{T} \sin \left(\theta_{T}-\sigma_{d}\right)-v_{R} \sin \left(\theta_{R}-\sigma_{d}\right)
\end{aligned}
$$

This is a simple two-dimensional differential system. This system is highly nonlinear, and the closed form solution is difficult in general. The kinematics equations (18) take into account the linear velocities and the orientation angles of the robot and the target and also the line of sight angle, which is an important geometric quantity. It is also possible to write the kinematics equations by taking the observer point as a reference point. This allows us to write

$$
\begin{aligned}
\dot{r}_{R}^{b} & =v_{R} \cos \left(\theta_{R}-\sigma_{R}^{b}\right) \\
r_{R}^{b} \dot{\sigma}_{R}^{b} & =v_{R} \sin \left(\theta_{R}-\sigma_{R}^{b}\right)
\end{aligned}
$$

and

$$
\begin{aligned}
\dot{r}_{T}^{b} & =v_{T} \cos \left(\theta_{T}-\sigma_{T}^{b}\right) \\
r_{T}^{b} \dot{\sigma}_{T}^{b} & =v_{T} \sin \left(\theta_{T}-\sigma_{T}^{b}\right)
\end{aligned}
$$

The distances $r_{R}^{b}$ and $r_{R}^{b}$ are measured from point $O_{b}$ and $\left(\sigma_{R}^{b}, \sigma_{T}^{b}\right)$ are measured from the reference line to the lines of sight $\left(\mathbf{L}_{\mathbf{R}}^{\mathbf{b}}, \mathbf{L}_{\mathbf{T}}^{\mathbf{b}}\right)$ as shown in Fig. 2(b). The navigation law which allows the robot to reach the moving goal is discussed in the next section.

\section{NAVIGATION STRATEGY}

The principle of the line of sight law is known in the aerospace community ([30]-[32]). The notion of observer used here is equivalent to the notion of ground control station. However to the best of our knowledge, the position of the ground station is always near the initial position of the pursuer. Whereas in this 
paper, there exists an infinite number of possibilities for the position of the observer, which are in accordance with assumption (H4). Our strategy is based on a variant of the line of the sight guidance law, which integrates the kinematics equations of the robot and the target and geometric rules. Here, the robot aims to reach its moving goal by matching the rate of turn of line of sight angle with the rate of turn of the target's line of sight angle measured from the stationary observer. This can be expressed as follows:

$$
\dot{\sigma}_{R}^{b}=\dot{\sigma}_{T}^{b}
$$

By integrating system (21), we get

$$
\sigma_{R}^{b}(t)-\sigma_{R}^{b}\left(t_{0}\right)=\sigma_{T}^{b}(t)-\sigma_{T}^{b}\left(t_{0}\right)
$$

From assumption (H4) (under which we have $\sigma_{R}^{b}\left(t_{0}\right)=$ $\left.\sigma_{T}^{b}\left(t_{0}\right)\right)$, it results that

$$
\sigma_{R}^{b}(t)=\sigma_{T}^{b}(t)
$$

This means that the control strategy is characterized by equal line of sight angles for the robot and the target. As a result, the robot lies always on the line of sight joining the target and the observer. The lines of sight $\mathbf{L}_{\mathbf{R}}^{\mathbf{b}}$ and $\mathbf{L}_{\mathbf{T}}^{\mathbf{b}}$ are on the same ray, which means that the distance robot-target under the control law is simply

$$
r_{d}=r_{T}^{b}-r_{R}^{b}
$$

and the line of sight angles for the robot and the target are equal to the line of sight robot-target (since $\mathbf{L}_{\mathbf{R}}^{\mathbf{b}}$ and $\mathbf{L}_{\mathbf{T}}^{\mathbf{b}}$ are on the same ray)

$$
\sigma_{R}^{b}=\sigma_{T}^{b}=\sigma_{d}
$$

Equation (21) allows us to write

$$
\frac{v_{R} \sin \left(\theta_{R}-\sigma_{d}\right)}{r_{R}^{b}}=\frac{v_{T} \sin \left(\theta_{T}-\sigma_{d}\right)}{r_{T}^{b}}
$$

from which we get the control input for the robot steering angle

$$
\theta_{R}=\sin ^{-1}\left(\frac{v_{T} r_{R}^{b}}{r_{T}^{b} v_{R}} \sin \left(\theta_{T}-\sigma_{d}\right)\right)+\sigma_{d} .
$$

Let

$$
k_{1}^{b}=\frac{r_{R}^{b}}{r_{T}^{b}}, \quad k_{2}=\frac{v_{R}}{v_{T}}
$$

where $k_{1}^{b}$ is the range ratio, where the ranges are measured from point $O_{b} ; k_{2}$ is the velocity ratio satisfying $k_{2}>1$ under assumption (H1). Note that $k_{1}^{b}$ is not constant during the navigation. The navigation strategy aims to match the positions of the target and the robot, which means that $r_{R}^{b} \rightarrow r_{T}^{b}$, and $k_{1}^{b}=1$ at the interception time. Thus, if $r_{R}^{b}\left(t_{0}\right)<r_{T}^{b}\left(t_{0}\right)$ then $k_{1}^{b} \leq 1$ in the time interval $t \in\left[t_{0}, t_{f}\right]$ (during the navigation process). We can also put

$$
k^{b}=\frac{k_{1}^{b}}{k_{2}}
$$

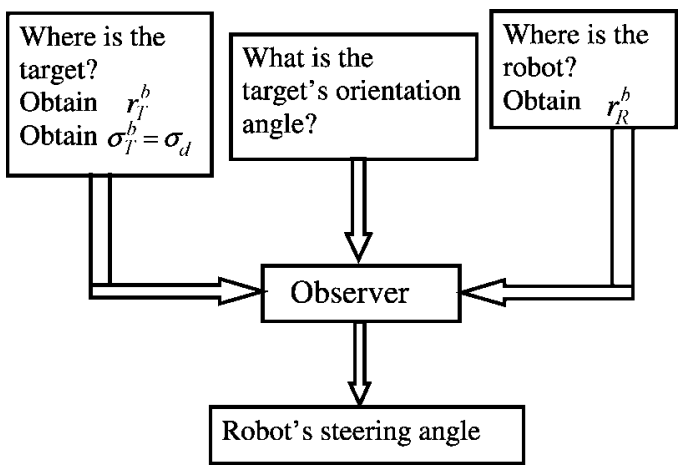

Fig. 3. Block diagram illustrating the guidance law strategy.

The control law (27) can be written as

$$
\theta_{R}=\sin ^{-1}\left(k^{b} \sin \left(\theta_{T}-\sigma_{d}\right)\right)+\sigma_{d}
$$

This equation is constrained by the following condition:

$$
k^{b} \leq 1
$$

Under assumption (H1) and assumption (H4) (from which we get $k_{1}^{b} \leq 1$ for $t \leq t_{f}$ ), we have $k^{b}<1$ in the time interval $t \in\left[t_{0}, t_{f}\right]$. Therefore, constraint (31) is satisfied.

The constraint on the initial positions given by $r_{R}^{b}\left(t_{0}\right)<$ $r_{T}^{b}\left(t_{0}\right)$, allows us to accomplish the navigation-tracking task with the minimum value of the robot's linear velocity, that is $v_{R}>v_{T}$. In some situations, for example, when at time $t_{0}$ the target is on the straight line between the observer and the robot, the control law requires higher values for the velocity ratio in order to keep $k^{b} \leq 1$. For example, if $k_{1}^{b}=c_{0}$, then (30) makes sense when $k_{2} \geq c_{0}$; which means that higher values for the velocity ratio are required for higher values of $c_{0}$. This problem is easily avoided when $r_{R}^{b}\left(t_{0}\right)<r_{T}^{b}\left(t_{0}\right)$. The second constraint resulting from $(\mathrm{H} 4)$, from which $\sigma_{R}^{b}\left(t_{0}\right)=\sigma_{R}^{b}\left(t_{0}\right)$ allows to elaborate a rigorous mathematical analysis of the control law, which is not possible in the general case. The generalization to other positions of the observer is an interesting topic for future investigation. This allows us to consider more than one observer in the workspace.

It is important to note that the control strategy for the robot steering angle depends on the ranges $r_{R}^{b}$ and $r_{T}^{b}$. This can be seen from (30) (since $k^{b}$ depends on $r_{R}^{b}$ and $r_{T}^{b}$ ). As a result, the steering angle of the robot depends on the coordinates of the observer point. Different paths for the robot are obtained for different observers. The control law can be seen as a three-point navigation law, since, in addition to the robot and the moving target, a stationary observer is also considered. In this case, the robot receives orders from the stationary observer. The observer collects the necessary information and sends the control command to the robot. The block diagram illustrating the guidance process is shown in Fig. 3.

An illustration of the control strategy is shown in Figs. 4 and 5. The robot aims to reach another robot moving in a vertical line (parallel to the y-axis). In Fig. 4, the observer is located at the origin, while in Fig. 5, the observer is located at point $(-10,0)$. 


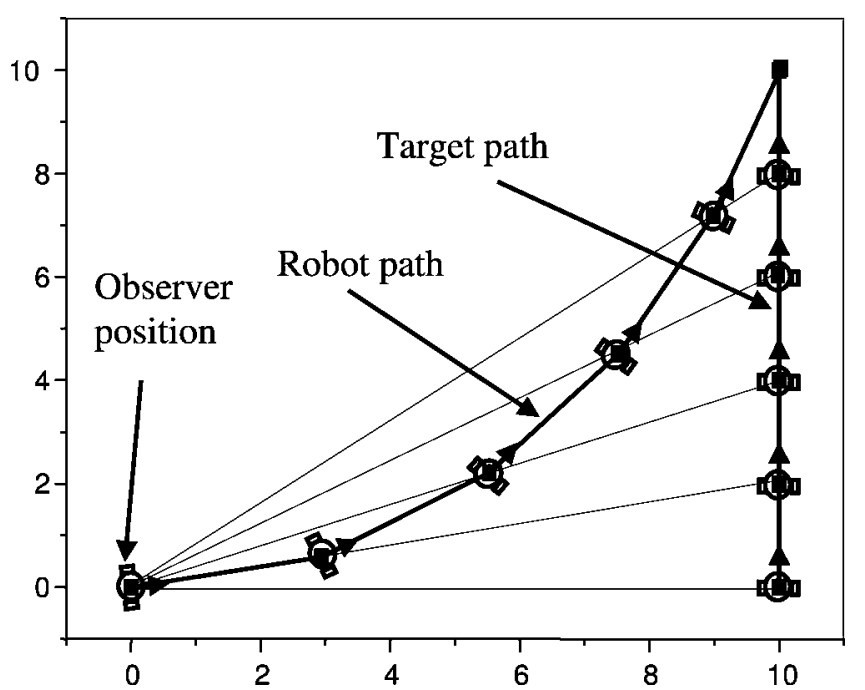

Fig. 4. Robot path for an observer situated at $(0,0)$, which is the initial position of the robot.

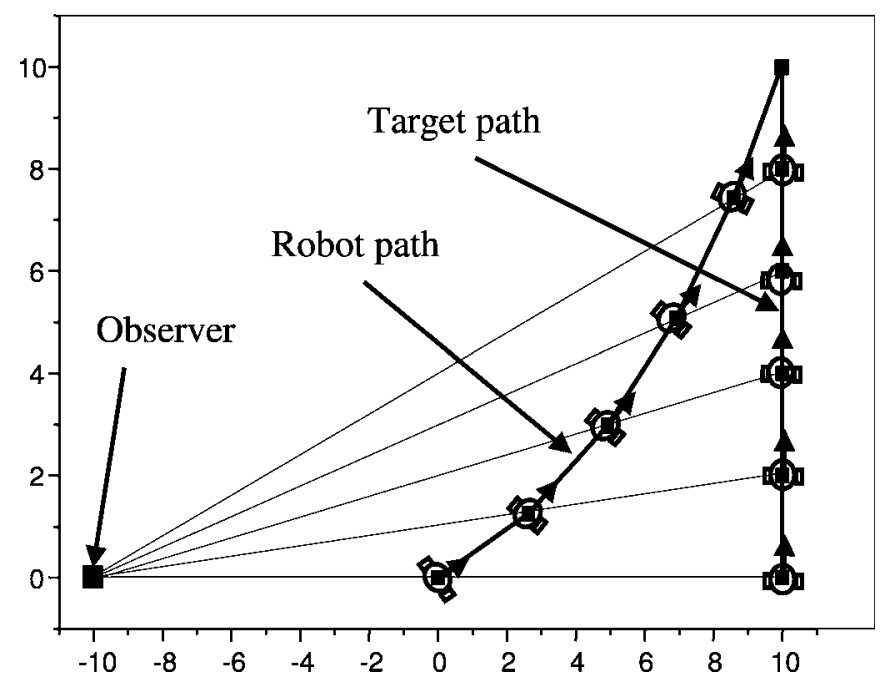

Fig. 5. Robot path for an observer situated at $(-10,0)$, the robot initial position is at $(0,0)$.

Recall that the velocity of the moving goal seen by the robot along and across the line of sight robot-target is given by

$$
\begin{aligned}
\dot{r}_{d} & =v_{T} \cos \left(\theta_{T}-\sigma_{d}\right)-v_{R} \cos \left(\theta_{R}-\sigma_{d}\right) \\
r_{d} \dot{\sigma}_{d} & =v_{T} \sin \left(\theta_{T}-\sigma_{d}\right)-v_{R} \sin \left(\theta_{R}-\sigma_{d}\right) .
\end{aligned}
$$

Thus, under the line of sight guidance law with an observer located at $O_{b}$, the relative distance between the robot and the target varies as follows:

$$
\dot{r}_{d}=v_{T} \cos \left(\theta_{T}-\sigma_{d}\right)-v_{R} \cos \left(\sin ^{-1}\left(k^{b} \sin \left(\theta_{T}-\sigma_{d}\right)\right)\right) .
$$

In a similar way, we get for the line of sight angle rate

$$
\begin{aligned}
r_{d} \dot{\sigma}_{d} & =v_{T} \sin \left(\theta_{T}-\sigma_{d}\right)-v_{R} k^{b} \sin \left(\theta_{T}-\sigma_{d}\right) \\
& =\left[v_{T}-v_{R} k^{b}\right] \sin \left(\theta_{T}-\sigma_{d}\right) .
\end{aligned}
$$

Our main result concerning the navigation using the line of sight guidance law and the interception of the moving goal is expressed in the following proposition.
Proposition 1: Under the control strategy given by (27), and the assumptions made previously on the position of the observer point and the velocity superiority of the robot, the robot reaches successfully its moving goal.

Proof: In order to prove that the robot reaches its moving goal, we proceed by proving that the relative range $r_{d}(t)$ between the robot and its goal is a decreasing function, that is $\dot{r}_{d}<0$. Recall that under the assumptions made previously on the position of the guidance reference point and the velocity superiority of the robot, we have $k^{b}<1$ for $t \in\left[t_{0}, t_{f}\right]$.

Let

$$
\begin{aligned}
& v_{R \|}=v_{R} \sin \left(\theta_{R}-\sigma_{d}\right) \\
& v_{T \|}=v_{T} \sin \left(\theta_{T}-\sigma_{d}\right)
\end{aligned}
$$

where $v_{R \|}$ is the robot's radial velocity and $v_{T \|}$ is the target's radial velocity. The range rate is given by

$$
\dot{r}_{d}=v_{T \|}-v_{R \|}
$$

The proof is based on the following remarks.

1) The inverse sine function maps the domain $[-1,1]$ into the range $[-(\pi / 2),(\pi / 2)]$, and since $k^{b}<1$, we have

$$
\sin ^{-1}\left(k^{b} \sin \left(\theta_{T}-\sigma_{d}\right)\right) \in\left(-\frac{\pi}{2}, \frac{\pi}{2}\right) \text {. }
$$

2) The cosine function of $x$ when $x \in(-(\pi / 2),(\pi / 2))$ is always positive.

3) From 1) and 2), we have

$$
\cos \left(\sin ^{-1}\left(k^{b} \sin \left(\theta_{T}-\sigma_{d}\right)\right)\right)>0 .
$$

Equation (38) implies that under the line of sight navigation strategy, the robot's radial velocity is always positive. By using (38), it is possible to write

$$
\cos \left(\sin ^{-1}\left(k^{b} \sin \left(\theta_{T}-\sigma_{d}\right)\right)\right)=\sqrt{1-\left(k^{b} \sin \left(\theta_{T}-\sigma_{d}\right)\right)^{2}} .
$$

The equation for the relative range becomes

$$
\dot{r}_{d}=v_{T} \cos \left(\theta_{T}-\sigma_{d}\right)-v_{R} \sqrt{1-\left(k^{b} \sin \left(\theta_{T}-\sigma_{d}\right)\right)^{2}}
$$

which can be reduced to

$\dot{r}_{d}= \pm v_{T} \sqrt{1-\left(\sin \left(\theta_{T}-\sigma_{d}\right)\right)^{2}}-v_{R} \sqrt{1-\left(k^{b} \sin \left(\theta_{T}-\sigma_{d}\right)\right)^{2}}$.

Depending on the sign of $v_{T \|}$, we have the following cases.:

First case: the moving goal is approaching: $\theta_{T}-\sigma_{d} \in$ $((\pi / 2),(3 \pi / 2))$, and

$$
\cos \left(\theta_{T}-\sigma_{d}\right)=-\sqrt{1-\left(\sin \left(\theta_{T}-\sigma_{d}\right)\right)^{2}}<0 .
$$

In this case, it is obvious that $\dot{r}_{d}<0, \forall \theta_{T}, \forall \sigma_{d}$.

Second case: the moving goal is escaping: $\theta_{T}-\sigma_{d} \in$ $(-(\pi / 2),(\pi / 2))$, and

$$
\cos \left(\theta_{T}-\sigma_{d}\right)=\sqrt{1-\left(\sin \left(\theta_{T}-\sigma_{d}\right)\right)^{2}}>0 .
$$

Since $k^{b}<1$, we get

$$
\sqrt{1-\left(\sin \left(\theta_{T}-\sigma_{d}\right)\right)^{2}}<\sqrt{1-\left(k^{b} \sin \left(\theta_{T}-\sigma_{d}\right)\right)^{2}}
$$

and because assumption (H1) states that $v_{R}>v_{T}$, we have $\dot{r}_{d}<0$ under the navigation law, $\forall \theta_{T}, \forall \sigma_{d}$. 
Third case: the target is moving with zero radial velocity: $\theta_{T}-\sigma_{d}= \pm(\pi / 2)$. In this case, it is clear that

$$
\dot{r}_{d}=-v_{R \|}<0 .
$$

Note that, the quantity $v_{T \|}-v_{R \|}$ is smaller in the case of approaching targets than in the case of escaping targets, which means that the interception is faster in the case of approaching targets.

The next result discusses the robot path in a special case.

Proposition 2: If $\theta_{T}$ is constant, and the observer is far away from the robot's initial position, that is $r_{d}$ is much smaller than $r_{R}^{b}$ or $r_{T}^{b}$, then the robot moves in a straight line.

Proof: Since $r_{d}$ is negligible compared to $r_{R}^{b}$ or $r_{T}^{b}$, we have $k_{1}^{b} \simeq 1$, and the control input for the robot orientation angle is

$$
\theta_{R} \simeq \sin ^{-1}\left(k_{2} \sin \left(\theta_{T}-\sigma_{d}\right)\right)+\sigma_{d} .
$$

This equation is obtained from

$$
v_{R} \sin \left(\theta_{R}-\sigma_{d}\right) \simeq v_{T} \sin \left(\theta_{T}-\sigma_{d}\right) .
$$

By combining (47) with the second equation in system (32), we get

$$
r_{d} \dot{\sigma}_{d} \simeq 0
$$

which means that $\sigma_{d}$ becomes constant when the choice of $O_{b}$ satisfies $r_{R}^{b}\left(t_{0}\right) \gg r_{d}\left(t_{0}\right)$. From (46), we see that $\theta_{R}$ is constant since $\theta_{T}, \sigma_{d}$ and $k_{2}$ are constant. This means that the robot moves in a straight line.

\section{A. Adaptive Line of Sight Guidance}

It is possible to change the position of the observer during the navigation process. This may become necessary when the robot is distant from the observer, and communication between the robot and the observer becomes difficult. In this approach, for each time interval $t \in\left[t_{i}, t_{i+1}\right], t_{i+1} \leq t_{f}$, a different observer point is used. One solution is to choose the position of the robot at a given time $t_{i}$ as the new observer for navigation at time $t>t_{i} \leq t_{f}$.

\section{B. Heading Regulation}

In many situations, the application of the line of sight guidance law to the robot is not straightforward, mainly because of the robot kinematics constraint (e.g., rolling without slipping constraint). To solve this problem, it is possible to use a heading regulation in order to drive the robot to an orientation angle that satisfies

$$
\theta_{R}\left(t_{0}\right)=\sin ^{-1}\left(k^{b}\left(t_{0}\right) \sin \left(\theta_{T}\left(t_{0}\right)-\sigma_{d}\left(t_{0}\right)\right)\right)+\sigma_{d}\left(t_{0}\right) .
$$

Different techniques from classical control theory can be used for this purpose.

\section{Tracking Without Interception}

In some situations, the aim is not to reach the moving goal, but to track it and keep it in a visibility zone. In this case, a second law for the robot speed is derived to keep the relative distance robot-target constant or above a given value. Clearly, constant distance between the robot and the goal corresponds to $\dot{r}_{d}=0$, and therefore, the control law for the robot speed to keep constant distance with the moving goal can be derived based on the range rate equation as follows:

$$
\dot{v}_{R}=-a\left(v_{R}-v_{R}^{*}\right)
$$

with

$$
v_{R}^{*}=v_{T} \frac{\cos \left(\theta_{T}-\sigma_{d}\right)}{\cos \left(\theta_{R}-\sigma_{d}\right)}
$$

for $\theta_{R} \neq \sigma_{d}$ and $a>0$. From (27), we have $\theta_{R} \neq \sigma_{d}$ unless $\theta_{T}=\sigma_{d}$. The case where $\theta_{R}=\theta_{T}=\sigma_{d}$ corresponds to the pure pursuit. Clearly, under (50), $v_{R}$ asymptotically tracks $v_{R}^{*}$.

\section{Implementation}

The control law given in (30) gives the quantities required by the control loop for the implementation of our strategy. Range sensors are required to measure the distances observer-target and observer-robot. This task can be accomplished using LIDAR or SONAR sensors [37]. The linear velocities can be obtained using velocity sensors such as Doppler LIDAR for example, or simply by taking the time derivative of the ranges. Since the observer is stationary, the velocity measured from the observer is the same as the velocity in the global frame of reference. The orientation and the line of sight angles can be obtained using orientation sensors. A communication system between the robot and the observer can be implemented and used by the robot to send its state to the observer, and receive the observer's commands. So, in order to implement the line of sight strategy, the system must keep track of the state of the moving goal, given by $\left(x_{T}, y_{T}, v_{T}, \theta_{T}\right)$ in our case. Estimation and tracking filters can be very useful to accomplish this task. Kalman filter techniques are among the most used for dynamic state estimation and tracking applications. These techniques are used in different fields such as missile guidance and ship navigation. Kalman filter uses two parallel cycles for state estimation: 1) state estimation and 2) state covariance estimation. The final state estimation is found from the predicted state, innovation and Kalman gain. Various sophisticated Kalman filters can be combined with the line of sight control strategy to enhance the navigation process by providing good estimate of the target position and velocity to the control loop.

\section{IN THE PResence of ObSTACles}

In the presence of obstacles, the navigation problem becomes more difficult, since reaching the moving goal consists of two tasks: 1) tracking the moving object and 2) obstacle avoidance. A combination between the line of sight guidance law and obstacle avoidance algorithms is necessary. The robot's navigates in two modes: tracking mode and obstacle avoidance mode. This yields a combination between global path planning and local obstacle avoidance to perform safe navigation among obstacles and reach the moving goal. It is clear that reaching a moving target is a real-time task. The robot starts tracking its moving goal using the line of sight guidance law. When an obstacle is detected within a specific distance from the robot, the obstacle avoidance algorithm is activated, and the robot deviates from its 
nominal path in order to avoid the obstacle. After the obstacle is passed, the robot returns to the navigation mode under the line of sight guidance law.

\section{A. Obstacle Avoidance Mode}

The workspace $W$ is cluttered up with $N$ stationary obstacles $B_{i}, i \in\{1, \ldots, N\}$. The aim of the robot is to accomplish $P_{R}\left(t_{f}\right)=P_{T}\left(t_{f}\right)$ with $P_{R}(t)$ collision-free. It must then satisfy

$$
P_{R}(t) \cap B_{i}=\emptyset \text {. }
$$

Only obstacles within a specific distance from the robot are considered. These obstacles lie in the robots active region, denoted by $X \subseteq W$. The robot free space is denoted by $C_{f r e e}$.

For obstacle avoidance, the method used is based on approximate cell decomposition. Note that various local obstacle avoidance methods can be used in combination with the line of sight guidance law. We choose the approximate cell decomposition for its simplicity and possibility to be combined with the line of sight guidance law. The robot's free space is represented as a collection of cells of rectangular shape. The use of such algorithm requires the discretization of the robot's kinematics equations under the line of sight control strategy. Here, for the discretization we use Euler's algorithm. The discretization on the time interval $\left[t_{0}, t_{f}\right]$ requires that the interval $\left[t_{0}, t_{f}\right]$ be partitioned into steps denoted by $n \in\{1, \ldots, K\}$. The derivative of the state variable $z$ with respect to time is approximated by

$$
\frac{d z}{d t}=\lim \frac{z(t+\Delta t)-z(t)}{\Delta t}, \quad \Delta t \rightarrow 0
$$

with $z(t)=z_{n}, z(\Delta t+t)=z_{n+1}$.

A rectangular decomposition $D$ of the robot's active region $X$ is a collection of rectangles $r_{i}, i=1, \ldots, M$ such that:

1) $X$ is equal to the union of $r_{i}, X=\cup_{i=1}^{M} r_{i}$;

2) The interiors of $r_{i}$ 's do not intersect, $\operatorname{int}\left(r_{i}\right) \cap \operatorname{int}\left(r_{j}\right)=$ $\emptyset, \forall i \in\{1, \ldots, M\}, \forall j \in\{1, \ldots, M\}, i \neq j$.

Each $r_{i}$ is called a cell in the robot's active region; it can be identified as:

1) Full: an obstacle $B_{i}$ fully covers the cell $r_{i}, r_{i} \subseteq B_{k}$.

2) Empty: the interior of the cell does not intersect with the obstacle, $r_{i} \cap B_{k}=\emptyset, \forall i \in\{1, \ldots, M\}, \forall k \in$ $\{1, \ldots, N\}$.

3) Mixed: $r_{i}$ is neither full nor empty.

Under the discrete model, the robot moves according to the following equations:

$$
\begin{aligned}
x_{R}(n+1) & =x_{R}(n)+\Delta t \cos \theta_{R}(n) \\
y_{R}(n+1) & =y_{R}(n)+\Delta t \sin \theta_{R}(n) \\
\theta_{R}(n) & =\sigma_{d}(n)+\sin ^{-1}\left(k^{b} \sin \left(\theta_{T}(n)-\sigma_{d}(n)\right)\right)
\end{aligned}
$$

with $k^{b}=\left(v_{T} r_{R}^{b}(n) / v_{R} r_{T}^{b}(n)\right)$, where $r_{T}^{b}(n)$ and $r_{T}^{b}(n)$ are the distances from the observer to the robot and the target at discrete time $n$, respectively. After the cell decomposition of the active region, the following algorithm is used to accomplish the task of tracking the target.

\section{B. Algorithm}

(1) Determine $\left(x_{R}(n+1), y_{R}(n+1)\right)$ according to the line of sight guidance law. This can be accomplished by using (54).

(2) Does $\left(x_{R}(n+1), y_{R}(n+1)\right)$ fall in a free cell? yes: move to $\left(x_{R}(n+1), y_{R}(n+1)\right)$; increase $n$ by 1 and go to (1).

- no: move to the nearest free cell from the position $\left(x_{R}(n+1), y_{R}(n+1)\right)$, increase $n$ by 1 and go to (1).

(3) Stop when the target is reached.

This algorithm allows a combination between navigation mode using the line of sight guidance law and the obstacle avoidance mode. In the next section, we simulate various scenarios.

\section{SimULATION}

We developed a simulation software using the object-oriented design paradigm. Numerous scenarios, including the scenarios shown in this section, can be generated using the software. We consider various scenarios, where the robot aims to reach a moving goal in both cases of presence and absence of obstacles. Both accelerating and nonaccelerating targets are considered. A comparison is carried out, especially with respect to the position of the observer. We assume that the speed, the distance and the time are without units for simplicity. In our simulation, it is assumed that, initially, (49) is satisfied, and thus heading regulation is not necessary. For the simulation of all considered scenarios, the observer is chosen such that $(\mathrm{H} 4)$ is satisfied.

\section{A. Obstacle-Free Workspace}

1) Case 1: Non-Accelerating Target: The target is moving in a straight line, with an orientation angle $\theta_{T}=0$. The speed of the target is $v_{T}=2$, the velocity ratio is $k_{2}=1.25$. The initial position of the target is $(20,20)$, and the initial position of the robot is $(0,0)$. The aim in this example is to illustrate the robot navigation toward the moving goal and also compare the paths obtained for different observers. We consider three cases: $O_{b}(0,0), O_{b}(-30,-30)$, and $O_{b}(-300-300)$. The paths of the target and the robot are depicted in Fig. 6, where the robot is navigating under the line of sight guidance law. The interception points are also different. The robot's path becomes more curved when $O_{b}$ approaches the initial position of the robot. Note that the path is a straight line when $O_{b}$ is located at $(-300,-300)$. This means that $\theta_{R}$ is constant. This case illustrates the property stated in proposition 2. Table I shows the position of the target and the robot for different observers, at different discrete times.

2) Case 2: Target Moving in a Circle: Here, we consider a target moving in a circle and starting at $(50,50)$, and the robot is initially at $(0,0)$. Two scenarios are illustrated in Fig. 7 , where the target moves clockwise and counterclockwise. The paths and positions of the robot and the target at different discrete times are shown in Fig. 7. In both cases the robot reaches its goal successfully. Tables II and III show the position of the target 


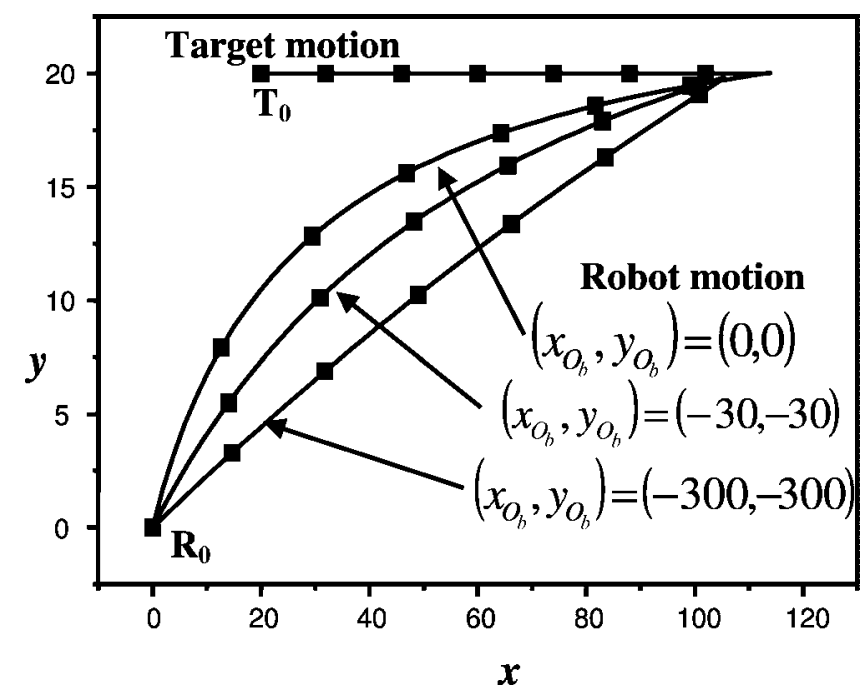

Fig. 6. Robot paths for tracking a target moving in a straight line for different positions of the observer.

TABLE I

TARget and Robot Positions at Discrete Times for the SCEnario of CASE 1. THe OBSERVER's Positions ARe $O_{B 1}(0,0), O_{B 2}(-30,-30)$, $O_{B 3}(-300,-300)$

\begin{tabular}{c|c|c|c|c}
\hline $\mathbf{t}$ & Target & $\mathrm{R}, O_{b 1}$ & $\mathrm{R}, O_{b 2}$ & $\mathrm{R}, O_{b 3}$ \\
\hline 0 & $(20,20)$ & $(0,0)$ & $(0,0)$ & $(0,0)$ \\
\hline 5 & $(32,20)$ & $(13,8)$ & $(14,5)$ & $(15,3)$ \\
\hline 12 & $(46,20)$ & $(30,13)$ & $(31,10)$ & $(32,7)$ \\
\hline 19 & $(60,20)$ & $(47,16)$ & $(48,13)$ & $(49,10)$ \\
\hline 26 & $(74,20)$ & $(64,17)$ & $(66,16)$ & $(66,13)$ \\
\hline 33 & $(88,20)$ & $(82,19)$ & $(83,18)$ & $(83,16)$ \\
\hline 40 & $(102,20)$ & $(99,19)$ & $(101,19)$ & $(101,19)$
\end{tabular}

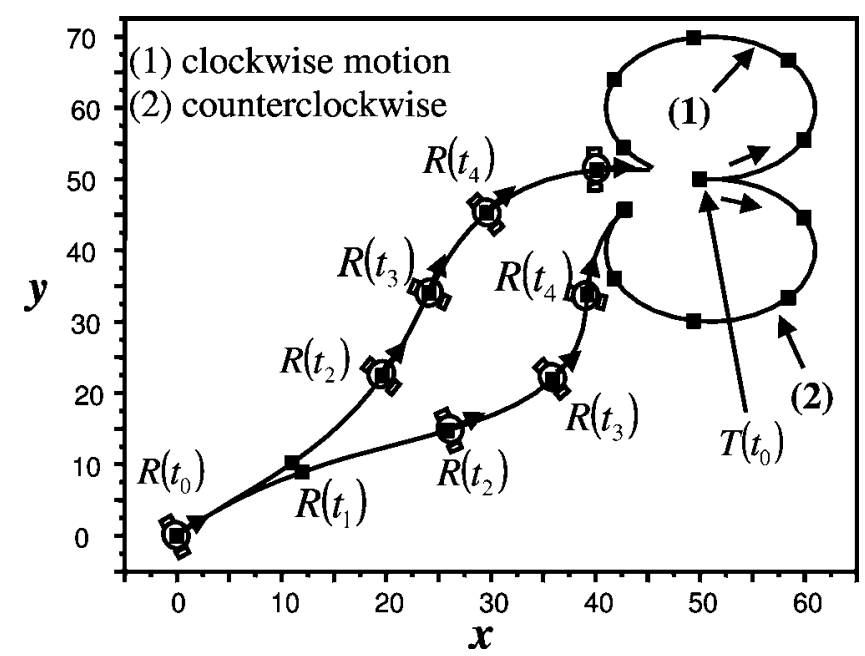

Fig. 7. Robot path for a target moving in a circle.

and the robot at different discrete times for the target performing clockwise and counterclockwise motions, respectively.

3) Case 3: Accelerating Target: Here we consider three scenarios. For the first scenario, illustrated in Fig. 8, the initial position for the target is $(150150)$, and $(0,0)$ for the robot. The paths of the robot and the target are shown in Fig. 8, where it is possible to compare between the path of robot obtained for an observer point located at $(0,0)$ (the initial position of the robot) and $(-300,-300)$. The robot reaches the moving goal in both cases, but at different positions and times. Table IV shows the
TABLE II

TARgET AND Robot Positions AT Discrete TIMES FOR THE SCENARIO of CASE 2. TARGET MOVING ClOCKWISE

\begin{tabular}{c|c|c}
\hline time & Target & Robot \\
\hline 0 & $(50,50)$ & $(0,0)$ \\
\hline 5 & $(59.9,44.6)$ & $(12,8.9)$ \\
\hline 11 & $(58.5,33.4)$ & $(25.8,14.7)$ \\
\hline 16 & $(49.4,30.1)$ & $(35.9,21.9)$ \\
\hline 21 & $(41.8,36.0)$ & $(39.2,33.8)$ \\
\hline 26 & $(42.7,45.6)$ & $(42.8,45.7)$ \\
\hline
\end{tabular}

TABLE III

TARgEt AND RoBot Positions at Discrete TIMES FOR THE SCENARIO of CASe 2. TARget Moving Counterclockwise

\begin{tabular}{c|c|c}
\hline time & Target & Robot \\
\hline 0 & $(50,50)$ & $(\mathbf{0 , 0 )}$ \\
\hline 5 & $(59.9,55.4)$ & $(11,10.2)$ \\
\hline 11 & $(58.5,66.7)$ & $(19.6,22.4)$ \\
\hline 16 & $(49.4,69.8)$ & $(24.1,34.1)$ \\
\hline 21 & $(41.8,64)$ & $(29.7,45.3)$ \\
\hline 26 & $(42.7,54.4)$ & $(40.2,51.2)$ \\
\hline
\end{tabular}

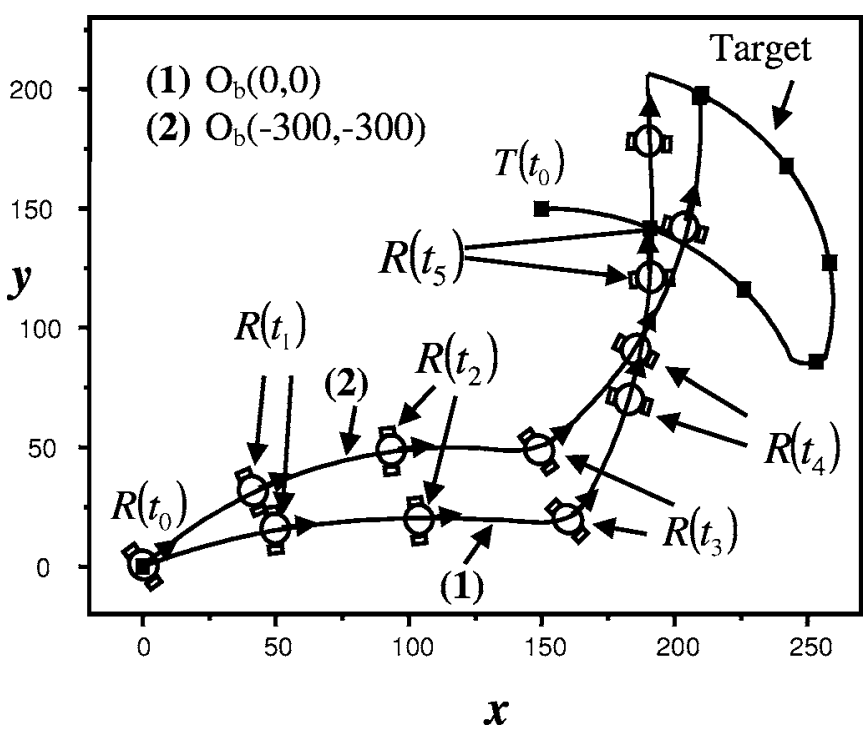

Fig. 8. Robot path for two different observers, target accelerating.

TABLE IV

TARGET AND Robot Positions at Discrete TIMES For THE SCENARIO OF CASE 3 FOR OBSERVERS SITUATED AT $O_{b 1}(0,0), O_{b 2}(-300,-300)$

\begin{tabular}{c|c|c|c}
\hline time & Target & Robot, $O_{b 1}$ & Robot, $O_{b 2}$ \\
\hline 0 & $(150,150)$ & $(0,0)$ & $(0,0)$ \\
\hline 20 & $(190,142)$ & $(42,31.2)$ & $(50.2,15.2)$ \\
\hline 42 & $(226,116)$ & $(94,48.3)$ & $(105,20.2)$ \\
\hline 64 & $(253,856)$ & $(149,50.3)$ & $(160,20.3)$ \\
\hline 86 & $(258,127)$ & $(185,91)$ & $(182,69.3)$ \\
\hline 108 & $(242,168)$ & $(205,142)$ & $(191,124)$ \\
\hline 130 & $(210,198)$ & $(209,197)$ & $(191,178)$ \\
\hline
\end{tabular}

position of the target and the robot for both observers at discrete times. For the second and the third scenarios, the target is performing a more difficult motion, which combines circular and sinusoidal motions.

- For the scenario of Fig. 9, the robot starts from point $(0,0)$, and the target starts from point $(95,95)$, the observer is situated at point $(-100,-100)$. 


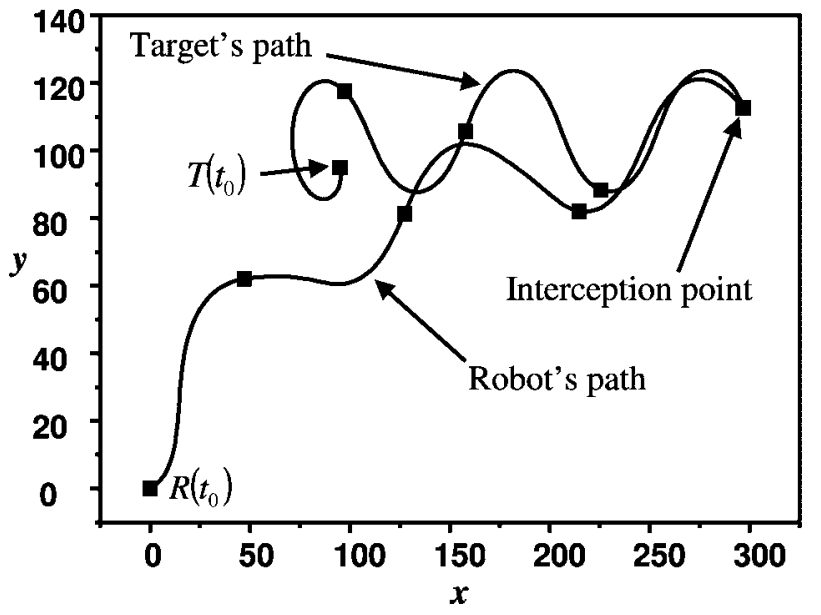

Fig. 9. Robot path for an accelerating target, observer situated at $(-100$, $-100)$.

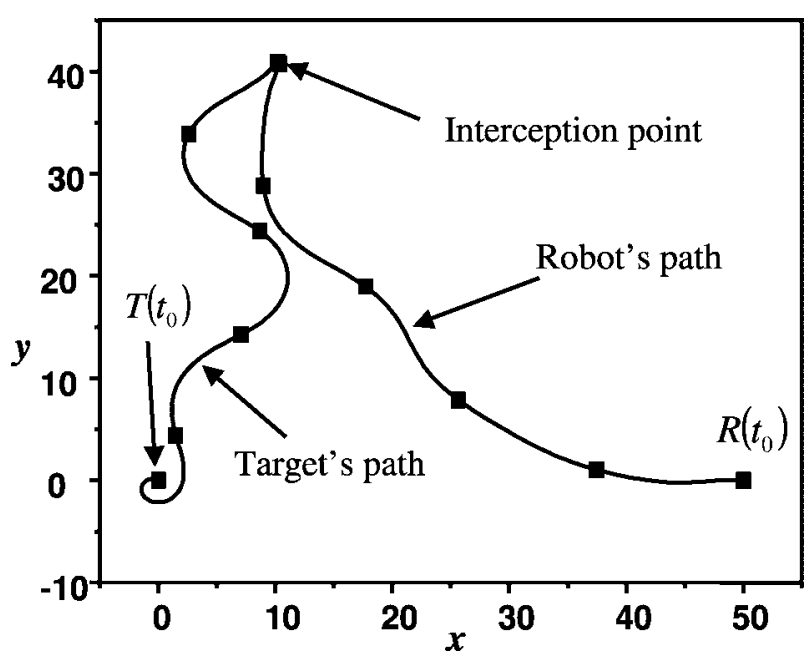

Fig. 10. Robot path for an accelerating target, observer situated at $(0,0)$.

TABLE V

TARget AND Robot Positions at Discrete Times FOR THE SCENARIO OF FIG. 9

\begin{tabular}{c|c|c}
\hline time & Target & Robot \\
\hline 0 & $(\mathbf{9 5}, 95)$ & $(0,0)$ \\
\hline 40 & $(97.5,117.4)$ & $(47.2,62)$ \\
\hline 80 & $(157.7,105.6)$ & $(127.3,81.3)$ \\
\hline 125 & $(225.6,88.27)$ & $(214.8,82)$ \\
\hline 168 & $(297,112.8)$ & $(296.7,112.5)$ \\
\hline
\end{tabular}

- For the scenario of Fig. 10, the robot starts from point $(50,0)$, and the target starts from point $(0,0)$, the observer is situated at point $(0,0)$.

In both cases (as shown in Figs. 9 and 10), the robot reaches successfully the goal. The positions of the robot and the goal at discrete times are shown in Tables V and VI.

\section{B. Comparison With the Pursuit Law}

Our aim is to provide a brief comparison with the pursuit law for reaching the moving goal. The pursuit law models various vision and sensor-based control laws. According to [38], the pursuit is used by ants to move in a convoy. We consider two simple examples with $v_{T}=2, v_{R}=2.5$.
TABLE VI

TARget And Robot Positions at Discrete Times FOR THE SCENARIO OF FIG. 10

\begin{tabular}{c|c|c}
\hline time & Target & Robot \\
\hline 0 & $(0,0)$ & $(50,0)$ \\
\hline 25 & $(1.52,4.4)$ & $(37.5,1)$ \\
\hline 50 & $(7.1,14.25)$ & $(25.64,7.9)$ \\
\hline 75 & $(8.7,24.4)$ & $(17.7,19)$ \\
\hline 100 & $(2.63,33.9)$ & $(9,28.9)$ \\
\hline 119 & $(10.3,40.9)$ & $(10.39,40.8)$ \\
\hline
\end{tabular}

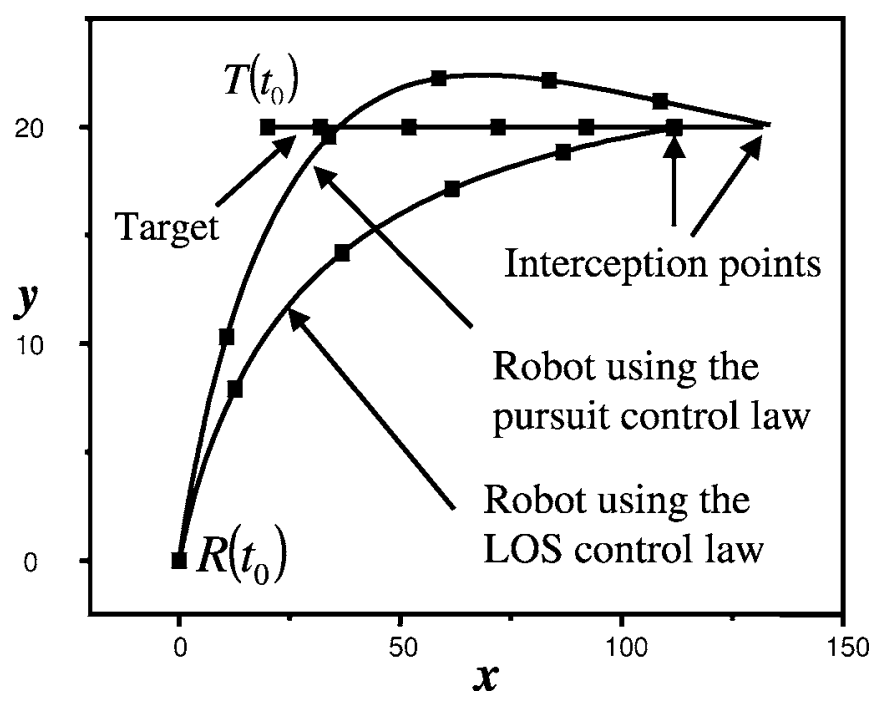

Fig. 11. Comparison with the pursuit, target moving in a straight line.

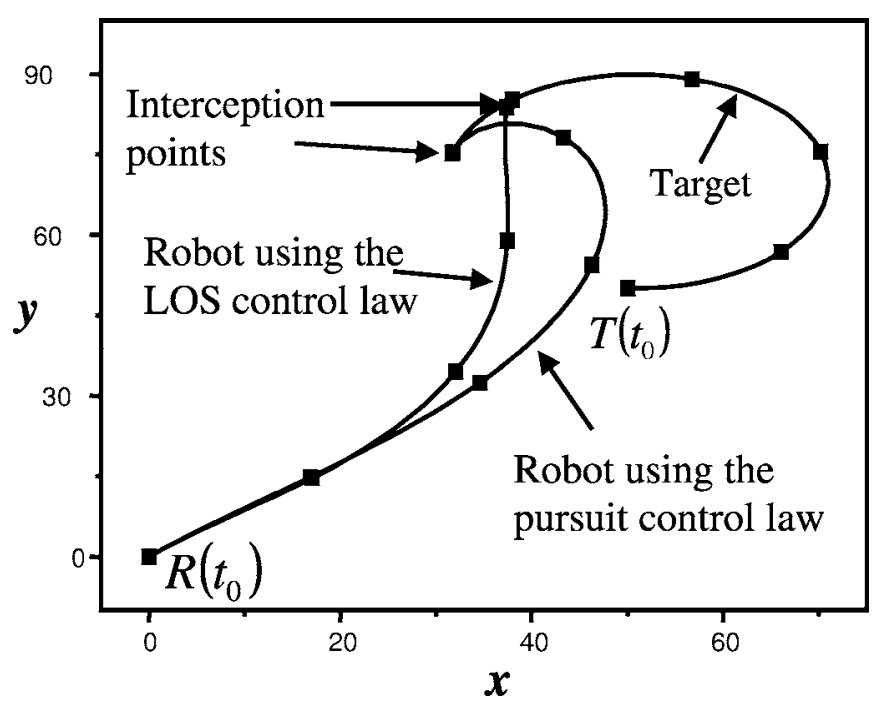

Fig. 12. Comparison with the pursuit, target moving in a circle.

Example 1: In this example, the target is moving in a straight line. The paths of the target and the robot under the line of sight and the pursuit control laws are shown in Fig. 11. The robot navigating under the line of sight reaches the goal before the pursuit. The interception times are $t_{f}=45$ and $t_{f}=55$ for the line of sight and the pursuit, respectively.

Example 2: The target is moving in a circle. The paths of the target and the robot under the line of sight and the pursuit control laws are shown in Fig. 12. Similarly to the previous example, the robot navigating under the line of sight reaches the goal before 
TABLE VII

COMPARISON With the PURSUIT, TARgET M OVING IN A StRaight LinE

\begin{tabular}{c|c|c|c}
\hline time & Target & Robot, pursuit & Robot, LOS \\
\hline $\mathbf{0}$ & $(20,20)$ & $(0,0)$ & $(0,0)$ \\
\hline 5 & $(32,20)$ & $(10.8,10.3)$ & $(12.7,7.9)$ \\
\hline 15 & $(52,20)$ & $(33.8,19.55)$ & $(36.9,14.9)$ \\
\hline 25 & $(72,20)$ & $(58.7,22.2)$ & $(61.7,17.2)$ \\
\hline 35 & $(92,20)$ & $(83.7,22.1)$ & $(86.7,18.8)$ \\
\hline 45 & $(112,20)$ & $(108.6,21.2)$ & $(111.7,20)$ \\
\hline
\end{tabular}

TABLE VIII

COMPARISON With THE PURSUIT, TARgET MOVING IN A CirCle

\begin{tabular}{c|c|c|c}
\hline time & Target & Robot, pursuit & Robot, LOS \\
\hline 0 & $(50,50)$ & $(\mathbf{0}, \mathbf{0})$ & $(\mathbf{0}, \mathbf{0})$ \\
\hline 8 & $(\mathbf{6 6 , 5 6 . 8 )}$ & $(\mathbf{1 6 . 9 , 1 4 . 9 )}$ & $(17,14.7)$ \\
\hline 18 & $(70.2,75.5)$ & $(34.6,32.4)$ & $(32.1,34.5)$ \\
\hline 28 & $(56.7,89.1)$ & $(46.3,54.3)$ & $(37.4,58.8)$ \\
\hline 38 & $(38,85.1)$ & $(43.3,78.2)$ & $(37.9,84.8)$ \\
\hline 44 & $(31.7,75.2)$ & $(31.7,75.3)$ & \\
\hline
\end{tabular}

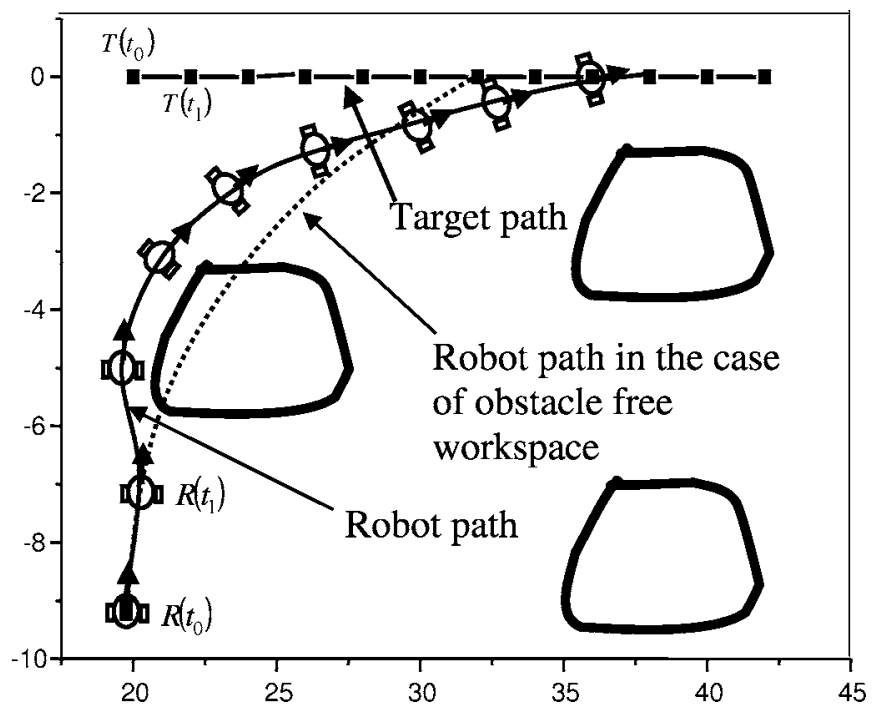

Fig. 13. Robot path in the presence of obstacles, target moving in a straight line.

the pursuit. The interception times are $t_{f}=38$ and $t_{f}=44$ for the line of sight and the pursuit, respectively. Also, the pursuit results in a more curved path.

The position of the target and the robot navigating under the line of sight and the pursuit are shown in Tables VII and VIII for the scenarios of example 1 and example 2, respectively.

\section{In the Presence of Obstacles}

Simulation of the navigation procedure in the presence of obstacles is shown in Figs. 13 and 14. The target moves in a straight line in the case of the Fig. 13. The robot changes its nominal path one time in order to avoid an obstacle. In the case of Fig. 14, the robot changes its nominal path two times. To return to the navigation mode, an adaptive line of sight strategy is used, where the last position of the robot in the obstacle avoidance mode is used as the new observer. In both cases, the robot reaches its moving goal successfully. For comparison, we also plotted in the same figures the paths of the robot in the case of obstacle free workspace in dashed lines. The position of the target and

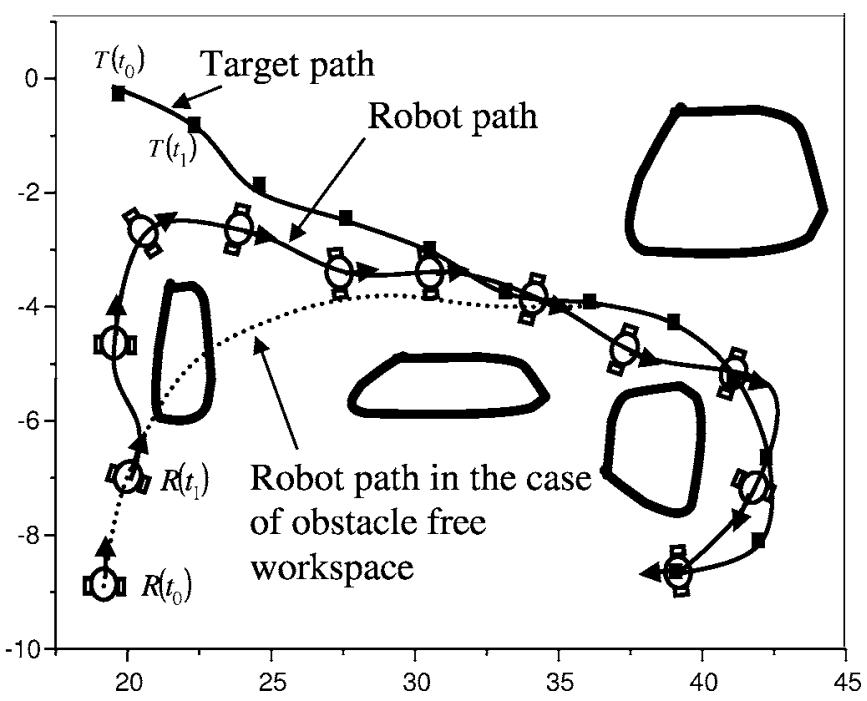

Fig. 14. Robot path in the presence of obstacles, target accelerating.

TABLE IX

Target And Robot Positions at Discrete Times in the Presence of OBSTACLES, TARGET MOVING IN A STRAIGHT LiNE

\begin{tabular}{c|c|c}
\hline time & Target & Robot \\
\hline 0 & $(20,0)$ & $(19.8,-9.25)$ \\
\hline 1 & $(22,0)$ & $(20.2,-7.2)$, \\
\hline 2 & $(24,0)$ & $(19.8,-5)$ \\
\hline 3 & $(26,0)$ & $(21.25,-3.1)$ \\
\hline 4 & $(28,0)$ & $(23.7,-2)$ \\
\hline 5 & $(30,0)$ & $(26.6,-1.3)$ \\
\hline 6 & $(32,0)$ & $(30,-0.9)$ \\
\hline 7 & $(34,0)$ & $(32.6,-0.5)$ \\
\hline 9 & $(36,0)$ & $(35.9,0)$ \\
\hline
\end{tabular}

TABLE $\mathrm{X}$

TARGET AND RoBOt POSITIONS AT DISCRETE TIMES IN THE PRESENCE OF OBSTACles, TARgET ACCELERATING

\begin{tabular}{c|c|c}
\hline time & Target & Robot \\
\hline 0 & $(19.7,-\mathbf{0 . 2 5 )}$ & $(19.5,-9)$ \\
\hline 1 & $(22.5,-\mathbf{0 . 7 5 )}$ & $(20,-7)$ \\
\hline 2 & $(24.7,-2)$ & $(19.4,-4.5)$ \\
\hline 3 & $(27.5,-2.4)$ & $(20.5,-2.7)$ \\
\hline 4 & $(30.5,-3)$ & $(23.8,-2.6)$ \\
\hline 5 & $(33.3,-3.8)$ & $(27.5,-3.4)$ \\
\hline 6 & $(36.2,-4)$ & $(30.8,-3.4)$ \\
\hline 7 & $(38.8,-4.3)$ & $(34.4,-3.9)$ \\
\hline 8 & $(41.2,-5.25)$ & $(37.5,-4.67)$ \\
\hline 9 & $(42,-6.6)$ & $(41.2,-5.1)$ \\
\hline 10 & $(41.6,-8.1)$ & $(41.8,-7)$ \\
\hline 11 & $(39.2-8.7)$ & $(39.2,-8.7)$ \\
\hline
\end{tabular}

the robot navigating under the line of sight are shown in Tables IX and X for the scenarios of Figs. 13 and 14, respectively.

\section{CONCLUSION}

In this paper, we presented a method for robot's navigation toward a moving goal. The goal's maneuvers are not a priori known to the robot. Our method is based on the use of geometric rules combined with the kinematics equations of the robot and the moving goal, where polar kinematics models are derived and used. The method is robust, since methods based on geometric 
rules are well known to be robust. The navigation law states that the robot moves with the same line of sight angle rate as the goal measured from an observer point. The path traveled by the robot is different for different observers. The method is well justified mathematically, and is proven that under the suggested navigation law, the robot reaches successfully the moving goal. Unlike many other control laws in dynamic environment, our method is a real-time strategy. In the presence of obstacles, the strategy is combined with an obstacle avoidance algorithm. Simulation for various scenarios is carried out, which proves the efficiency of the method and the validity of our theoretical results.

\section{REFERENCES}

[1] O. Khatib, "Real-time obstacle avoidance for manipulators and mobile robots," Int. J. Robot. Res., vol. 5, no. 1, pp. 90-98, 1986.

[2] E. Rimon and D. Kopditschek, "Exact robot navigation using artificial potential functions," IEEE Trans. Robot. Autom., vol. 8, no. 5, pp. 501-517, Oct. 1992.

[3] S. Ge and Y. Cui, "New potential functions for mobile robot path planning," IEEE Trans. Robot. Autom., vol. 16, no. 5, pp. 615-619, Oct. 2000.

[4] J. Borenstein and Y. Koren, "Histogramic in-motion mapping for mobile robot obstacle avoidance," IEEE Trans. Robot. Autom., vol. 7, no. 4, pp. 535-539, Aug. 1991

[5] — , "The vector field histogram—fast obstacle avoidance for mobile robots," IEEE Trans. Robot. Autom., vol. 7, no. 3, pp. 278-288, Jun. 1991.

[6] F. Chaumette, "Images moments: a general and useful set of features for visual servoing," IEEE Trans. Robot., vol. 20, no. 4, pp. 713-723, Aug. 2004.

[7] G. DeSouza and A. Kak, "Vision for mobile robot navigation: a survey," IEEE Trans. Pattern Anal. Mach. Intell., vol. 24, no. 2, pp. 237-267, Feb. 2002.

[8] R. Luo and T. Chen, "Autonomous mobile target tracking system based on grey-fuzzy control algorithm," IEEE Trans. Ind. Electron., vol. 47, no. 4, pp. 920-931, Aug. 2000.

[9] T. Li, S. Chang, and W. Tong, "Fuzzy target tracking control of autonomous mobile by using infrared sensors," IEEE Trans. Fuzzy Syst., vol. 12, no. 4, pp. 491-501, Aug. 2004.

[10] W. Tong and T. Li, "Realization of two-dimensional target tracking problem via autonomous mobile robot using fuzzy sliding mode control," in Proc. IEEE Int. Conf. Industrial Electronics, Aachen, Germany, Aug. 1998, pp. 1158-1163.

[11] R. Stonier and M. Mohammadian, "Knowledge acquisition for target capture," in Proc. IEEE Int. Conf. Evolutionary Computation, Anchorage, AK, May 1998, pp. 721-726.

[12] T. Chen and R. Luo, "Mobile robot tracking using hierarchical grey-fuzzy motion decision-making method," in Proc. IEEE Int. Conf. Robotics and Automation, San Francisco, CA, Apr. 2000, pp. 2118-2123.

[13] R. Luo, T. Chen, and K. Su, "Target tracking using a hierarchical greyfuzzy motion decision-making method," IEEE Trans. Syst., Man, Cybern. A, Syst., Humans, vol. 31, no. 3, pp. 179-186, May 2001.

[14] I. Jeong and J. Lee, "Evolving fuzzy logic controllers for multiple mobile robots solving a continuous pursuit problem," in Proc. IEEE Int. Conf. Fuzzy Systems, Seoul, Korea, Aug. 1999, pp. 685-690.

[15] L. Sung-On, C. Young-Jo, H.-B. Myung, Y. Bum-Jae, and O. Sang-Rok, "A stable target-tracking control for unicycle mobile robots," in Proc. IEEE/RSJ Int. Conf. Intelligent Robots and Systems, Takamatsu, Japan, Oct. 2000, pp. 1822-1827.

[16] M. Adams, "High speed target pursuit and asymptotic stability in mobile robotics," IEEE Trans. Robot. Autom., vol. 15, no. 2, pp. 230-237, Apr. 1999.

[17] H. Yamaguchi, "A cooperative hunting behavior by mobile robot troops," Int. J. Robot. Res., vol. 18, no. 8, pp. 931-940, 1999.

[18] _ "A distributed motion coordination strategy for multiple nonholonomic mobile robots in cooperative hunting operations," Robot. Auton. Syst., vol. 43, pp. 257-282, 2003.
[19] S. Ge and Y. Cui, "Dynamic motion planning for mobile robots using potential field method," Auton. Robots, vol. 13, pp. 207-222, 2002.

[20] E. Marchand, P. Bouthemy, F. Chaumette, and V. Moreau, "Robust real-time visual tracking using a 2d-3d model-based approach," in Proc. IEEE Int. Conf. Computer Vision, Keryra, Greece, Sept. 20-27, p. 1999.

[21] M. Tsai, K. Chen, M. Cheng, and K. Lin, "Implementation of a real-time moving object tracking system using visual servoing," Robotica, vol. 21, pp. 615-625, 2003.

[22] L. Hsu and F. Lizarralde, "Robust adaptive visual tracking control: Analysis and experiments," in Proc. IEEE Int. Conf. Control Applications, Anchorage, AK, Sep. 2000, pp. 25-27.

[23] A. Cretual, F. Chaumette, and P. Bouthemy, "Complex object tracking by visual servoing on 2d image motion," in Proc. IEEE Int. Conf. Pattern Recognition, Brisbane, Australia, Aug. 1998, pp. 1251-1254.

[24] M. M. M. Brackstone, "Car-following: a historical review," Transport. Res. F, vol. 2, pp. 181-196, 1999.

[25] F. Chaumette, P. Rives, and B. Espiau, "Positioning of a robot with respect to an object, tracking it and estimating its velocity by visual sevoing," in Proc. IEEE Int. Conf. Robotics and Automation, Sacramento, CA, Apr. 1991, pp. 2248-2253.

[26] S. Feyrer and A. Zell, "Detection, tracking, and pursuit of humans with an autonomous mobile robot," in Proc. IEEE/RSJ Int. Conf. Intelligent Robots and Systems, IROS'99, Kyonglu, Oct. 1999, pp. 864-869.

[27] —_, "Tracking and pursuing persons with a mobile robot," in Proc. Int. Workshop on Recognition, Analysis and Tracking Faces and Gestures in Real-Time Systems, Los Alamitos, CA, Sept. 1999, pp. 83-88.

[28] J. Dias, C. Paredes, I. Fonseca, H. Araujo, J. Batista, and A. Almeida, "Simulating pursuit with machine experiments with robots and artificial vision," IEEE Trans. Robot. Autom., vol. 14, no. 1, pp. 1-17, Feb. 1998.

[29] B. Kim, D. Roh, J. Lee, K. Son, M. Lee, J. Choi, and S. Han, "Localization of a mobile robot using images of a moving target," in Proc. IEEE Int. Conf. Robotics and Automation, Seoul, Korea, May 2001, pp. 253-258.

[30] J. Kain and D. Yost, "Command to the line of sight guidance: stochastic optimal control problem," J. Spacecraft, vol. 14, no. 7, pp. 438-444, 1977.

[31] I. Ha, "Design of a close guidance law via feedback linearization," IEEE Trans. Aerosp. Electron. Syst., vol. 28, no. 1, pp. 51-63, Jan. 1992.

[32] D. Roddy, G. Irwin, and H. Wilson, "Optimal controllers for bank-toturn clos guidance," Proc. Inst. Elect. Eng., vol. 131, no. 4, pp. 109-116, 1984.

[33] C. D. Yang and C. Yang, "A unified approach to proportional navigation," IEEE Trans. Aerosp. Electron. Syst, vol. 33, no. 2, pp. 557-567, Apr. 1997.

[34] M. Mehrandezh, M. Sela, R. Fenton, and B. Benhabib, "Proportional navigation guidance for robotics interception of moving objects," $J$. Robot. Syst., vol. 17, no. 6, pp. 321-340, 2000.

[35] M. Mehrandezh, N. M. Sela, R. G. Fenton, and B. Benhabib, "Robotic interception of moving objects using an augmented ideal proportional navigation guidance techniques," IEEE Trans. Syst., Man, Cybern. A, Syst., Humans, vol. 30, no. 3, pp. 238-250, May 2000.

[36] F. Belkhouche and B. Belkhouche, "A method for robot navigation toward a moving goal with unknown maneuvers," Robotica, to be published.

[37] M. Adams, "Coaxial range measurement—current trends for mobile robotic applications," IEEE Sensors J., vol. 2, no. 1, pp. 2-13, Feb. 2002.

[38] A. Bruckstein, "Why the ant trails look so straight and nice," Math. Intelligencer, vol. 15, no. 2, pp. 59-62, 1993.

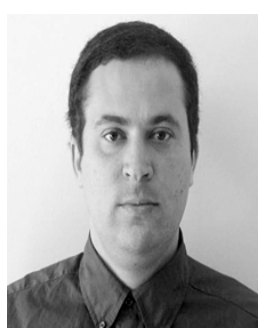

Fethi Belkhouche (M'01) received the B.S. degree in electrical engineering in 1997 and the M.S. degree in electronic physics in 2001, both from the University of Tlemcen, Algeria. He is currently working toward the Ph.D. degree in electrical engineering in the Department of Electrical Engineering and Computer Science, Tulane University, New Orleans, LA.

His research interests include guidance theory, robot navigation, robot cooperation and formation control, and linearization methods.

Mr. Belkhouche is a member of the AIAA. 


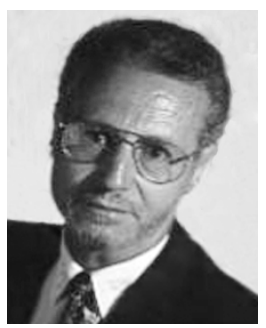

Boumediene Belkhouche (M'82) is a Professor of electrical engineering and computer science at Tulane University, New Orleans, LA. His research areas include motion planning for autonomous mobile robots and object-oriented modeling.

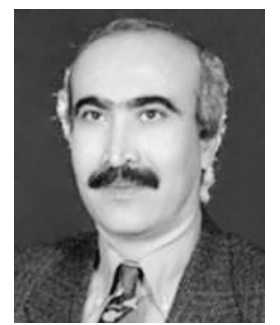

Parviz Rastgoufard (SM'01) received the B.S. degree in electrical engineering from State University of New York, Buffalo, in 1976 and the M.S. and Ph.D. degrees in systems science from Michigan State University, Ann Arbor, in 1978 and 1983, respectively.

He has been with the Department of Electrical Engineering and Computer Science, School of Engineering, Tulane University, New Orleans, LA, since July 1987. He serves as the Chair of the Department of Electrical Engineering and Computer Science and the holder of Energy Chair in Electric Power Engineering. His main research interests are large-scale electric transmission and generation systems planning, operation, and control in general and systems stability and security modeling, simulation, and analysis. 